\title{
Comparative Study of the Wind Codes: An Application to Forty-Six Storied Wall-Frame Structure
}

\author{
B. Kiriparan, J.A.S.C. Jayasinghe and U.I. Dissanayake
}

\begin{abstract}
Wind is a random movement of air particles in both time and space, which produces very complicated dynamic loading scenario on flexible structures like tall buildings. Modern tall buildings are becoming more slender, flexible, lightweight and irregular in shape due to revolution of associated technologies. Consequently, analysis of tall buildings considering complicated nature of wind loading and dynamic response of the structural system is an important role in design of tall buildings. Wind tunnel test is the most reliable tool for the estimation of dynamic wind loading on tall buildings. However, due to the cost and time involved, wind design codes are generally used during the preliminary design stage. Thus, understanding the background of dynamic wind loading and procedures adopted in wind design standards to represent the dynamic effects is vital to arrive at an efficient, safe and economical structural system during the preliminary design stage. This paper presents an overview on background of dynamic wind loadings and provisions of four international wind codes frequently referred to in Sri Lanka, British Standard (BS), European Standard (BS EN), Australian Standard (AS/NZS) and Standard of Architectural Institute of Japan (AIJ). Further, the concept of equivalent static load derived based on the "gust-factor" method adopted in most of the international wind design codes is discussed. At the end, a forty-six storied wall-frame structure was used as the numerical example for the explanation of dynamic wind loading and its influence on the structural design.
\end{abstract}

Keywords: Tall building; Wind code, Along wind response; Across wind response, Torsional wind responses

\section{Introduction}

Around the world, construction of tall buildings has been increasing over time to overcome the scarcity of land. Colombo, Sri Lanka's commercial capital, is one such very active metropolitan city in tall building development. Modern tall buildings are more slender, flexible, lightweight and irregular in shape due to the factors such as advanced technologies in construction, improved vertical transportation, choice of lightweight partitions over the traditional heavy masonry partitions and development of parametric architectural design philosophies. As a result, contemporary tall buildings are more vulnerable for the dynamic wind loading [1], [2] and [3].

Wind tunnel testing is a very reliable tool to investigate wind effects on such dynamically sensitive tall buildings. However, due to the associated cost and time, performing a wind tunnel test in the preliminary design stages may not be always viable. Thus, three existing code provisions of wind design standards and virtual wind tunnels (with the involvement of Computational Fluid Dynamics) are utilized in the preliminary design stage and then, it is verified through the wind tunnel tests at the final design stage. Due to the complex nature of wind and its interaction with the structure, several limitations and significant discrepancies are found between the international standards in prediction of wind effects on dynamically sensitive tall buildings [1], [4] and [5]. Thus, selection of most suitable wind design standard with understanding of its capabilities and the limitations is utmost important to make a precise estimation of wind induced responses. This will eventually assist the designer to arrive at a safe and optimum structural scheme during the conceptual design stage.

Eng. B.Kiriparan, C.Eng., MIE(Sri Lanka), B.Sc. Eng.

(Peradeniya), Department of Civil Engineering, Faculty of

Engineering, University of Peradeniya.

Email:kiriparan@gmail.com

(iD http://orcid.org/0000-0003-4633-7234

Eng.(Dr.) J.A.S.C. Jayasinghe, AMIE(Sri Lanka), B.Sc. Eng.

(Peradeniya), M.Eng. (AIT), Ph.D. (Tokyo), Senior Lecturer,

Department of Civil Engineering, Faculty of Engineering,

University of Peradeniya.

Email:supunj@pdn.ac.lk

(D) http://orcid.org/0000-0003-1054-9358

Eng.(Dr.) U.I. Dissanayake, C.Eng., MIE(Sri Lanka), B.Sc.

Eng.(Peradeniya), Ph.D. (Sheffield), Senior Lecturer,

Department of Civil Engineering, Faculty of Engineering,

University of Peradeniya.

Email:udissa@pdn.ac.lk

(D) $h$ ttp://orcid.org/0000-0001-5802-7239 
A clear understanding on the background of dynamic wind loading and application of provisions of wind design standards is vital in that regard. This paper intends to provide an overview of theoretical aspects of dynamic wind loading on tall buildings and demonstrate various provisions of existing wind design codes. The concept of equivalent static load derived based on the "gust-factor" method, adopted in most of the international wind design codes, is discussed. Important considerations in provisions of four international wind codes frequently referred to in Sri Lanka, British Standard (BS 6399-2:1997), European Standard (BS EN 1991-1-4:2005), Australian Standard (AS/NZS1170.2:2011) and Architectural Institute of Japan (AIJ: 2004), are also discussed. Further, wind loading responses of a standard 46 storied building tested in wind tunnel [6] are determined using these different codes/standards and a comparison is presented to explain the review. A detailed discussion on along wind, across wind and torsional responses are made. At the end, importance of selecting a suitable wind design approach during the preliminary design to predict dynamic wind effects and their impact on tall building design is emphasized.

\section{Characteristics of Wind}

Wind is a dynamic and random phenomenon. Fluctuation of the wind speed shown in Figures 1 and 2 illustrate the random nature of wind with both time and along building height, respectively.

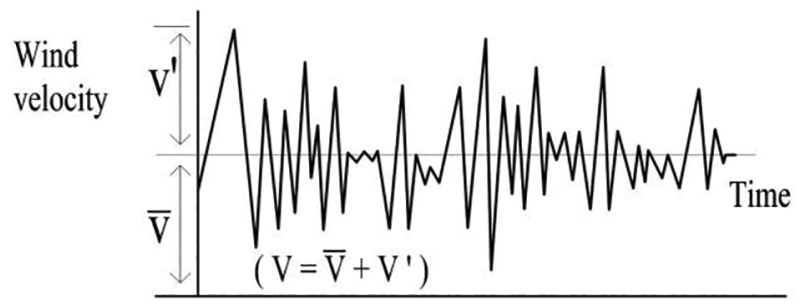

Figure 1 - Wind Speed Variation with Time

$$
\begin{aligned}
& \mathrm{V} \text { - Instantaneous wind speed } \\
& \mathrm{V}^{\prime} \text { - Fluctuating component } \\
& \bar{V} \text { - Mean component }
\end{aligned}
$$

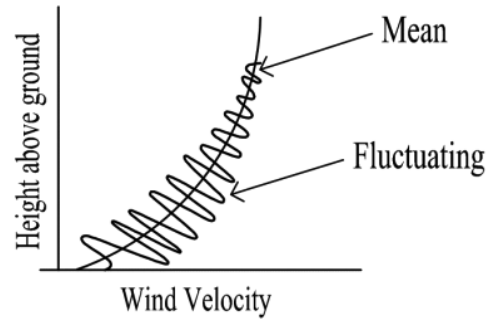

Figure 2 - Wind Speed Variation along Height

The dynamic and random nature of wind in both time and special characteristic is considered when describing wind speed. Terrain roughness has high influence in the variation of velocity profile over the height as shown in Figure 3. The frictional drag forces reduces gradually as the distance from earth surface increases; thus, velocity profile reaches a uniform value beyond a height referred to as gradient height. The region below this gradient height is known as atmospheric boundary layer. Depth of the boundary layer is dependent on terrain roughness and typically ranges from $250 \mathrm{~m}$ to $500 \mathrm{~m}$ [7].

Power law Eq. 1, logarithmic law Eq. 2 and Deaves and Harris (D\&H) model are three well established relationships adopted by different international codes to predict the wind velocity profile with height.

Power Law: $\frac{u}{u_{r}}=\left(\frac{z}{z_{r}}\right)^{\alpha}$

$u_{z}$ - wind speed at height $z$

$u_{r}$ - known wind speed at reference height $z_{r}$

$\alpha$ - Hellmann exponent for terrain category

Log Law: $u_{z}=\left(\frac{u_{*}}{k}\right)\left(\ln \left(\frac{z}{z_{0}}\right)-\varphi\left(\frac{z}{L_{*}}\right)\right)$

$u_{*}-$ friction velocity

$k=0.4-$ von Karman's constant

$z_{0}$ - roughness length

$L_{*}$ - Monin Obukhov length

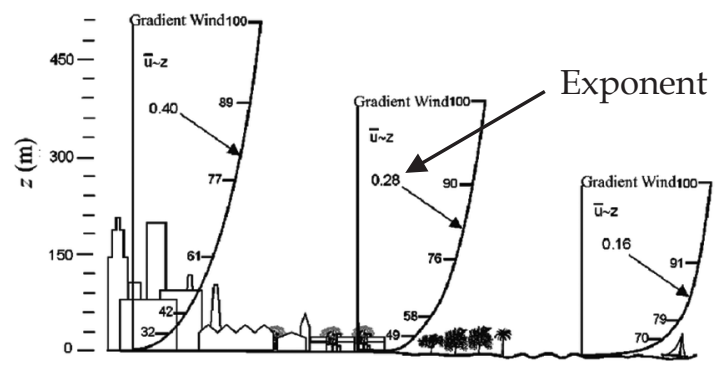

Figure 3 - Profile of Mean Wind Velocity Over Flat Terrain of Different Roughness 
Different number of terrain categories is adopted in each wind code as summarized in Table 1. Due to variation of roughness length and power exponent values adopted in each code, significant discrepancies are encountered in the prediction of velocity profile. Discussion on proposal for unified terrain categories for harmonization of these discrepancies can be found elsewhere [8], [9] and [10]. Further, a topography factor is incorporated in the wind codes to accommodate the variation of velocity profile and gradient height due to topography features such as hills and valleys.

Table 1 - Summary of Terrain Categories Defined in Various Wind Codes

\begin{tabular}{|c|c|c|}
\hline Standard & $\begin{array}{c}\text { Number of } \\
\text { terrains }\end{array}$ & Law \\
\hline BS 6399-2-1997 & 3 & D\&H \\
\hline BSEN1991-1-4:2005 & 5 & Log \\
\hline AS/NZS1170.2:2011 & 4 & Log \\
\hline AIJ-RLB-2004 & 5 & Power \\
\hline
\end{tabular}

Prevailing wind, seasonal wind and local wind are three major types of wind that are important in design of buildings. The mean velocities of prevailing wind fluctuating over a period of several months is referred as fluctuations. Rapid variations of local winds occurring over very short periods (every few seconds) are referred to as gusts. As a result, characteristics of prevailing and seasonal winds are grouped together while those of local winds are studied separately when determining wind loads. Theoretically, the mean wind velocity averaged over different averaging times should be determined by dominant extreme events, for example 3-seconds for thunderstorm downdrafts or out flows, 10 minutes for tropical cyclones or typhoons and 1 hour for extratropical gales. Though, mean velocities with different averaging time periods (3seconds, 10-minutes and 1-hour) are adopted in each wind code, a single averaging time is followed throughout a particular standard [12], [14], [15] and [16].

However, in the calculation of wind induced responses, all codes utilize a longer period such as 10 minutes or 1-hour as tabulated in Table 2 . In addition, the reference height $\left(h_{\text {ref }}\right)$ at which the gust loading factor and other parameters are calculated is different among the standards, as summarized in Table 2. The effect on the intermediate parameters during the calculation of gust loading factor and wind loads, due to these differences in averaging time and reference heights from one code to another, makes a simple comparison between the standards challenging. A conversion factor is generally utilized to make a balance among different averaging times in basic wind speed [5].

Table 2 - Averaging Time and Reference Heights

\begin{tabular}{|c|c|c|c|c|}
\hline \multirow{3}{*}{ Standard } & \multirow{2}{*}{\multicolumn{2}{|c|}{ Averaging time }} & \multirow{3}{*}{$\begin{array}{l}\text { Return } \\
\text { period }\end{array}$} & \multirow{3}{*}{$\begin{array}{c}\text { Ref. } \\
\text { height } \\
\left(h_{\text {ref }}\right)\end{array}$} \\
\hline & & & & \\
\hline & $\begin{array}{l}\text { Wind } \\
\text { speed }\end{array}$ & $\begin{array}{l}\text { Wind } \\
\text { response }\end{array}$ & & \\
\hline BS & 1-hr & 1-hr & 50 & $\mathrm{~h}$ \\
\hline BSEN & 10-min & 10-min & 50 & $0.6 \mathrm{~h}$ \\
\hline AS/NZS & 3-sec & 10-min & 50 & $\mathrm{~h}$ \\
\hline AIJ-RLB & 10-min & 10-min & 100 & $\mathrm{~h}$ \\
\hline
\end{tabular}

Statistical distributions of wind speeds and directions are more preferred over simple averages due to the random nature of wind loading [7]. Return periods of 50 years and 100 years are considered for the basic wind speeds as summarized in Table 2.

The wind speed is varying in each direction depending on the geographic location of the site. Dominant wind direction and relative orientation of the building are important factors influencing the wind loading on tall buildings. Based on wind climatic study of different regions, directional factors for varying angles are provided in wind codes to accommodate variation of wind speed with direction. Further, shielding multipliers are used in wind codes to incorporate shielding effect due to adjacent buildings. However, wind tunnel testing with different surrounding conditions will require to accurately interpret the interference effects such as funnelling. In all of the wind codes, design wind speed at specified height, $V_{d}$, is determined by factoring basic wind speed at reference height $V_{b}$ as shown in Eq. 3 .

$$
V_{d}=V_{b} S_{e x} S_{t o} S_{d i} S_{i m} S_{o t}
$$

where $S_{e x}$ is the velocity profile or exposure factor, $S_{t o}$ is the topography factor, $S_{d i}$ is the wind directionality factor, $S_{i m}$ is the building importance factor and $S_{o t}$ is a factor accounting for other things such as hurricane zone, shielding or mean recurrence interval (return period). Further, due to the very low viscosity (about one-sixteenth of water) any movement of air at speeds greater than $0.9-1.3 \mathrm{~m} / \mathrm{s}$ (2-3 $\mathrm{mph}$ ) is turbulent, causing particles of air to move randomly in all directions. There is no obvious correlation between the random fluctuations at the different heights. Turbulence intensities are proposed in the wind codes to accommodate this phenomenon. 


\section{Dynamic Wind Loading on Tall Buildings}

Wind applies dynamic forces on the structure when wind engulfs the structure. The response of a structure for applied dynamic wind loads depend on the dynamic characteristics of the structure. Amplified structural response can result from the dynamic loadings compared to that obtained from equivalent static loads. Fundamental natural frequency of vibration, $f_{0}$, of a structure is the most widely accepted parameter used to determine significance of dynamic response under wind loading. ASCE Standard [ASCE 7-10] classifies a structure as dynamically sensitive, or "flexible" if $f_{0}<1 \mathrm{~Hz}$, otherwise it is considered to be "rigid." This structural classification is broadly accepted as a reasonable boundary between dynamic and rigid behaviour [7].

Commonly, first mode of a tall building is associated with the lowest frequency and smooth monotonic deformation; the higher modes feature increasing frequency and inflection points. The dominant frequency of wind gusts is relatively low compared to the lowest natural frequency of building structures as shown in Figure 5, and mostly excites the lowest mode of vibration. Therefore, generally the lowest mode of vibration is only considered when examining dynamic response to wind. This applies separately to each of three components of response (sway in the $\mathrm{x}$ and $\mathrm{y}$ directions and twist about the vertical z-axis). This is in contrast to dynamic response to earthquakes, where the dominant excitation energy is in the frequency range of low-rise buildings or the higher modes of tall buildings. Hence, a large number of modes must generally be considered in seismic analysis. Further, earthquake load is applied only at a single point (on ground) rather than distributed over the height.

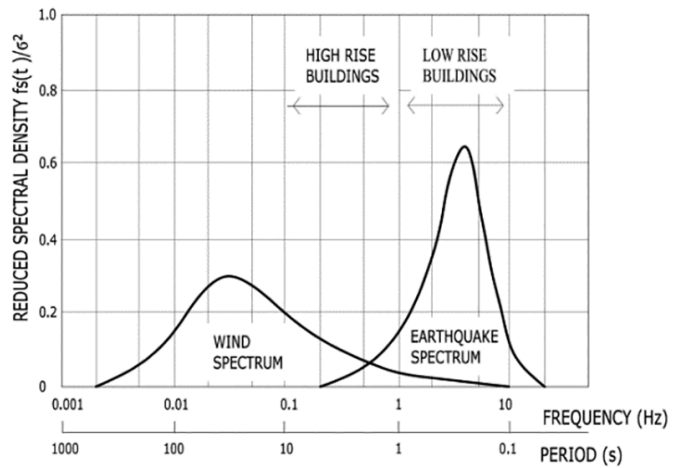

Figure 5 - Frequency Range of Structures Excited by Wind and Earthquakes [7]
Figure 6 illustrates a Single Degree of Freedom (SDOF) system that represents dynamic response of tall buildings. Mathematical idealization of the structure subjected to excitation $P(t)$ and the free body diagram illustrating how this external load is balanced by the internal spring force, damping force, and inertial force are shown in Figure 6 (a) and Figure $6(\mathrm{~b})$, respectively. The internal spring force may be regarded as the static-equivalent load, as applying this load statically to the system would result in the same spring force.

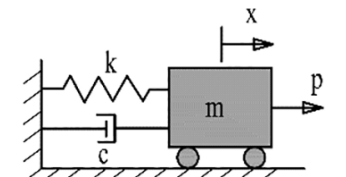

(a)

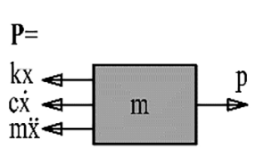

(b) $p=$ external (aerodynamic) load

$\mathbf{P}=$ internal (static equiv.) load

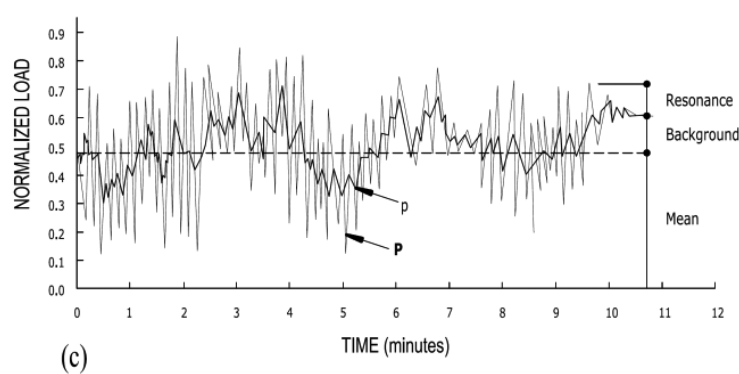

Figure 6 - Dynamic Response of an SDOF System: (a) Idealized System; (b) Free-Body Diagram Showing Balance of Forces and (c) Sample Excitation and Responses

If the excitation of the SDOF system is sinusoidal, i.e., with the equation of motion

$$
m \ddot{x}+c \dot{x}+k x=P_{0} \sin 2 \pi f t
$$

then the steady-state solution is $x(t)=$ $x_{0} \sin (2 \pi f t-\omega)$, where the instantaneous amplitude is well known to be,

$$
x(t)=\frac{P(t) / k}{\sqrt{\left[1-\left(\frac{f}{f_{0}}\right)^{2}\right]^{2}+\left[2 \varepsilon \frac{f}{f_{0}}\right]^{2}}}
$$

The magnitude of the internal response force, then, is conveniently expressed as

$$
P=|H(f)| \mathrm{P}
$$

where

$$
|H(f)|^{2}=\frac{1}{\left[1-\left(\frac{f}{f_{0}}\right)^{2}\right]^{2}+\left[2 \varepsilon \frac{f}{f_{0}}\right]^{2}}
$$


is generally regarded as the mechanical admittance.

Above equations (1) to (7) provide two important observations. Firstly, the internal response load to the external applied load depends only on the natural frequency and damping, as opposed to the mass or stiffness separately. Secondly, a non-dimensional function, $H(f)$, applies to any input and output parameters linearly related to the load, and also the output response could be conveniently written as a boldface version of the input excitation.

For example,

$$
X=|H(f)| x
$$

where $x$ would be the displacement under static application of the load $P$, and $X$ is the dynamic displacement.

The characteristic behaviour of $|H(f)|$ is presented in Figure 7. Resonance condition occurs in the buildings with very low damping if the excitation frequency is close to the natural frequency of the structure. This is the scenario in the flexible buildings under wind loadings. The function $|H(f)|$ describes the resonance condition with an amplification factor.

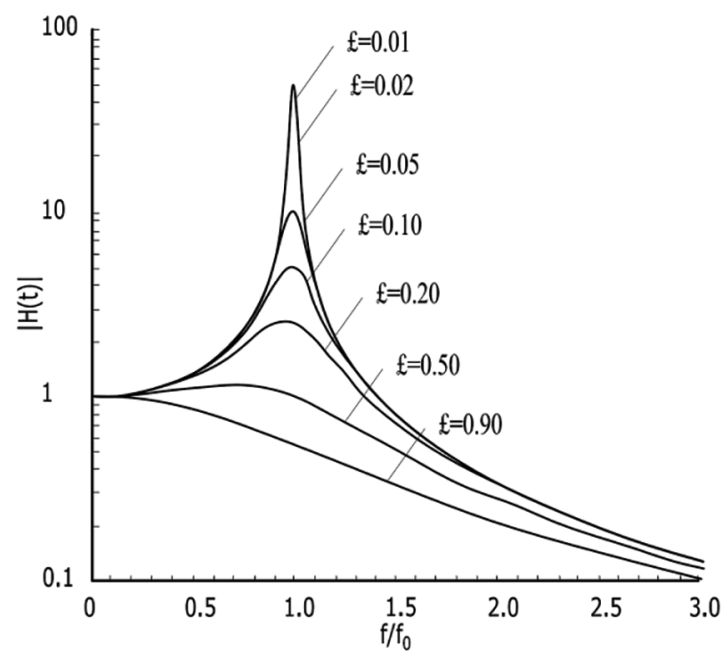

Figure 7 - SDOF Dynamic Amplification Factor

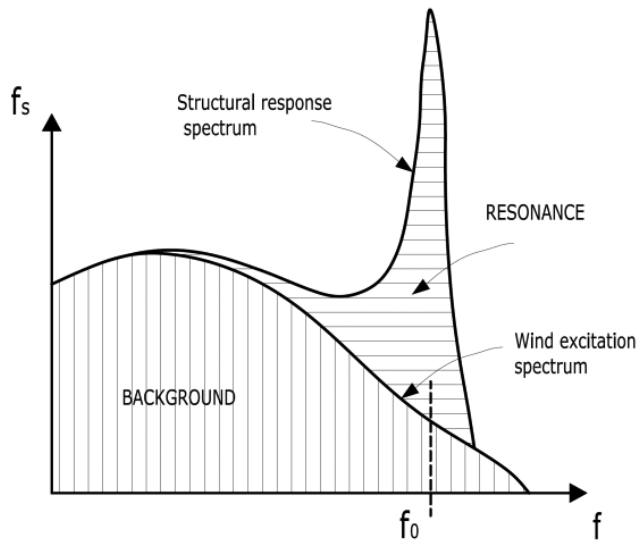

Figure 8 - Background and Resonance Contributions to the Response Spectrum

Wind excitation is not sinusoidal but a spectrum consisting of a random superposition of a broad range of frequencies. Unlike earthquake engineering, here the term "response spectrum", is mean square spectral density (spectrum) of the response (displacement, static-equivalent load or moment, or static-equivalent generalized load) and can be derived by weighting the mean square spectral density of the excitation as in Equation 9. Figure 8 illustrates the characteristics of excitation and response spectrum.

$$
S_{P}(f)=|H(f)|^{2} S_{P}(f)
$$

The above SDOF system equation can be equally applied to one mode of a Multiple Degree of Freedom (MDOF) system building simply by substituting the generalized load $P^{*}$ for the simple load $P . P^{*}$ is closely related to the response base moment, and with some adjustment for particular mode shapes, can lead to internal response forces applicable at each floor level [7].

In wind engineering, the response load is evaluated by super-positioning two components, background and resonance. The former is the quasi-static response that is equal to the external aerodynamic load and occurs when the natural frequency is extremely higher (or equivalently, to wind gusts having a frequency much lower than the natural frequency). The latter represents the additional amplification that is embedded in $|H(f)|$ and, for practical purposes, may be considered the dynamic response to those gusts having a duration close to the natural period of the structure. The mean square value of the fluctuating response is obtained by integrating the response spectrum: 


$$
\sigma_{P}^{2}=\int_{0}^{\infty}|H(f)|^{2} S_{P}(f) d f
$$

The peak response is defined by superimposing its mean value with the root-mean-square (rms) multiplied by a peak factor. This procedure is greatly facilitated by first obtaining the peak values of the background, and resonant portions are described below.

Since the background response is considered to be the load itself without resonant amplification, its $r m s$ value is simply $\sigma_{P}$. The spectrum of the resonant portion is then:

$$
S_{P, R}(f)=S_{P}(f)-S_{P}(f) \ldots
$$

as indicated in Figure 6. The rms value of the resonant portion is therefore

$$
\sigma_{P, R}=\sqrt{\sigma_{P}^{2}-\sigma_{P}^{2}}
$$

Under particular yet common conditions, this can be represented by the well-known white noise approximation, avoiding the integration indicated in Equation (10):

$$
\sigma_{P, R} \approx \sqrt{\frac{\pi}{4 \varepsilon} f_{0} S_{P}\left(f_{0}\right)}
$$

The peak value of the fluctuating response is now found by combining the background and resonant peaks using mean square addition, and adding the mean response:

$$
\hat{P}=\bar{P} \mp \sqrt{\left(g_{0} \sigma_{P}\right)^{2}+\left(g_{1} \sigma_{P, R}\right)^{2}}
$$

The peak background factor is generally taken as, approximately,

$$
g_{0}=3.5
$$

and the peak resonance factor is generally calculated from Davenport's formula [7]

$$
g_{1}=\sqrt{2 \ln f_{1} T}+\frac{0.577}{\sqrt{2 \ln f_{1} T}}
$$

where $f_{1}$ is the natural frequency of the first mode of vibration, and $T$ is the time period to which the peak value is referenced, generally taken as $1 \mathrm{hr}$.

This approach is adopted in all current wind design codes for the treatment of dynamic response of flexible buildings. Dynamic aspects are covered by introducing a "gust factor" to multiply the quasi-static loads utilized in a static analysis of the structure.

\section{Wind Effects on Tall Buildings}

Complicated flow patterns such as distortion of mean flow, flow separation, formation of vortices, and development of the wake are generated when wind engulf a structure. Large wind pressure fluctuations due to these effects can occur on the surface of a building and thus imposing very large aerodynamic loads on the structural system. Due to these fluctuating forces, building is intended to vibrate in rectilinear and torsional modes, as illustrated in Figure 9. The response of a structure subjected to these fluctuating wind loads is highly dependent on the nature of the aerodynamic forces and the dynamic characteristics of the building.

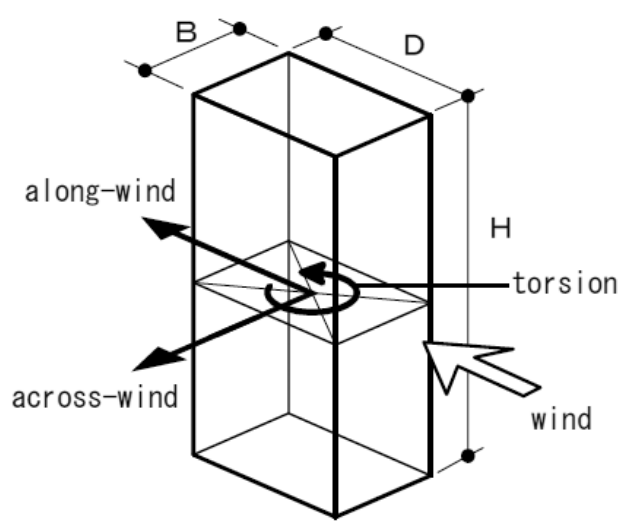

Figure 9 - Wind Effect on Tall Buildings

Due to the complex nature of wind, the design wind loading in a particular axis of the building can be governed when wind blows even in any other direction than the axis considered.

Figure 10 presents the variation of base moment, $M x$, of a symmetrical rectangular building for wind approaching in 360-degree range with constant wind speed. The mean, background and resonance responses of the building resulting from three natural frequencies of vibration are presented. Alongwind response is represented by wind at 0 and 180 degrees. Peak value of the along wind load is approximately twice the mean value. Due to the high turbulence, the building starts to experience a harmonic vortex shedding from buffeting when wind blows nearly in 90 and 270 degree directions. At this condition, the response is almost entirely dynamic. Due to the strong vortex, shedding across wind loading is observed to be even much higher than the along wind loading. 


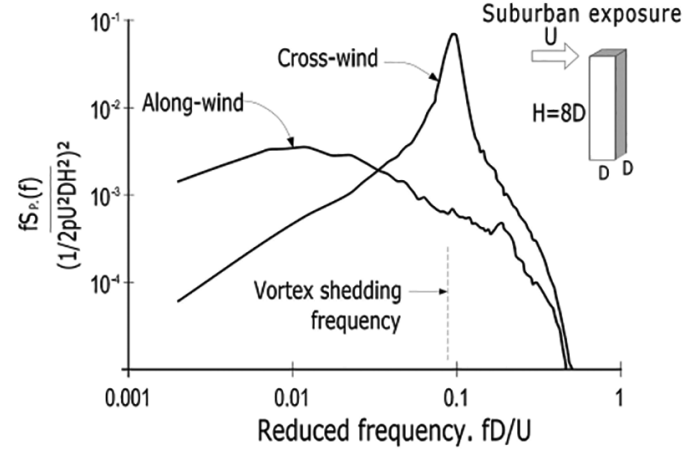

Figure 10 - Response Base Moment for a Tall Isolated Flexible Building Showing the Influence of Natural Frequency [7]

Excitation spectra for a tall and slender building obtained from a wind-tunnel model study are shown in Figure 11. The spectra illustrate that the resonant response of the building to the along wind loadings monotonically decreases with increase of natural frequency. Nevertheless, the vortexshedding phenomenon (illustrated in Figure 12) introduces a peak value at an intermediate frequency for cross wind loadings, thus extremely affects the resonant response. Strouhal number, $S_{t}$, is an important parameter to define the frequency of vortex shedding.

Strouhal number, $S_{t}=\frac{f_{s} D}{u}$

$f_{s}$ - vortex shedding frequency

$D$ - width or diameter of the structure

$u$ - wind speed

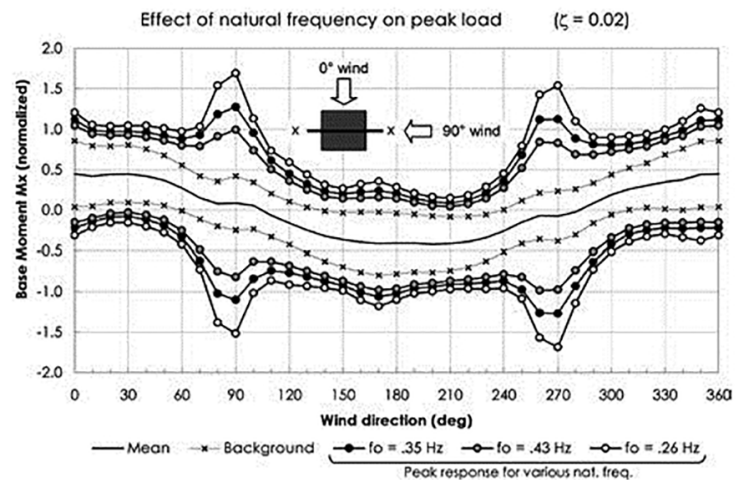

Figure 11 - Aerodynamic Load Spectra Measured on a Wind-Tunnel Model [7]

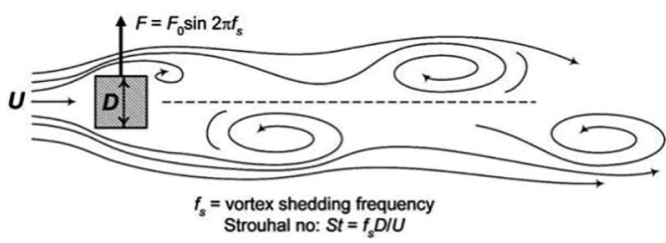

Generally, the shedding frequency falls below the natural frequency of most of the buildings. When the stiffness of the building is reduced and natural frequency is lowered this phenomenon will happen. The intensity of shedding, and therefore the rate at which load increases with decreased stiffness, is highly dependent on the building shape and the amount of turbulence in the approaching wind. Regular symmetrical shapes and smooth flows result in more intense shedding.

Dynamic response in torsion is another important consideration in tall building design. Torsion is induced due to variations and fluctuations in the wind velocity along the building face, which creates an unequal pressure distribution. Eccentricity between the instantaneous aerodynamic centre and the centre of building's rigidity induces torsional motions in the buildings. Greater torsional moments are developed when breadth of the building is larger, due to wind phenomenon such as turbulence buffeting or vortex shedding. Torsional effects become strong when first fundamental mode of the building is torsional mode.

It is very rare that, all three moment components, $M x, M y$ and $M z$, of a particular building achieving their peaks simultaneously at a specific wind direction. The load combinations are selected based on the concept of principal companion actions as shown in Figure 13. Establishing the design load cases to a building will be more complex when adding the $\mathrm{Mz}$ component in the action combinations as no load cases yield a governing case. Wind tunnel studies have adopted various techniques to find governing conditions, while building codes have attempted to address this recently.

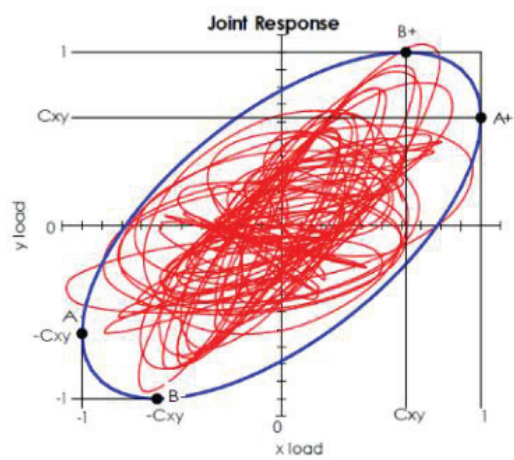

Figure 13 - Simultaneous Response of Two Base Load Components

Figure 12 - Vortex Shedding 


\section{Comparison of Wind Design Codes/ Standards}

Wind design standards are utilized to determine wind loading on tall buildings during the preliminary design stage. Many international design codes/standards are developed for the prediction of wind response of tall buildings. Although each standard is developed based on the same theoretical basis, discrepancies are observed between their estimates. Further, each standard imposes limitations for its application, based on the building shape, natural period and building height. These restrictions are imposed due to the complex nature of dynamic response of the building, as previously discussed in this paper. Consequently, the responses predicted from different codes deviate from one to another [4], [14], [15] and [16]. Hence, the wind design codes should be used with consideration to the constraints.

In order to understand the differences, provisions of four major international wind codes BS 6399-2:1997, S/NZS1170.2:2011, EN1991-1-4:2005 and AIJ RLB: 2004 were investigated in this paper. All four standards considered set out provisions for the prediction of along wind loading. Only AS/NZS1170.2:2011 and AIJ RLB: 2004 consist of provisions for prediction of across wind loading. Torsional moments are specified in the form of nominal eccentricities in AS/NZS1170.2:2011 and EN1991-1-4:2005 standards, whereas AIJ RLB: 2004 provides a detailed method for the calculation of torsional moment.

\subsection{Along-wind Loads}

The general expression for wind pressures $\left(p_{z}\right)$ on a building from all the codes/standards is:

$$
p_{z}=q_{z} G C_{p}
$$

where $q_{z}$ is the velocity pressure at height $\mathrm{z}, G$ is the gust effect factor and $C_{p}$ is the pressure coefficient. The loads are then determined by combining the wind pressures acting on the building surface multiplied by corresponding tributary areas. Moments are determined by multiplying the load at a given height by the corresponding height. Base shear forces and moments are then determined by the summation of the loads and moments at each level.
The velocity pressure $\left(q_{z}\right)$ can be generalized as:

$$
q_{z}=\frac{1}{2} \rho V_{d z}^{2}
$$

where $\rho$ is the air density and $V_{d z}$ is the design wind speed at height of interest as determined from Eq. 3. The gust effect factor $(G)$ for the codes/standards may be written in a general format as:

$$
G=\frac{G L F}{G_{q}}
$$

where GLF is the gust loading factor and $G_{q}$ is the gust factor for the wind velocity pressure. The term gust effect factor $(G)$ is used in ASCE 7-10, while it is defined differently in international codes/standards. For reference, AS/NZS describes it as dynamic response factor $\left(C_{d y n}\right)$ and EU as structural factor $\left(C_{s} C_{d}\right)$. GLF, originally defined by Davenport [11], is generally expressed as:

$$
G L F=1+r \sqrt{g_{B}^{2} B+g_{R}^{2} R}
$$

where $g_{B}$ and $g_{R}$ are the peak factors for background and resonant responses, respectively, $r$ is the turbulence intensity related variable, $B$ and $R$ are the background and resonant factors, respectively.

The gust factor for the wind velocity pressure in Eq. 17 is intended to compensate for the difference in averaging times, e.g., between basic wind velocity and wind-induced response calculation (Table 2).

$$
G_{q}=2 G_{v}-1=1+r \cdot g_{v}
$$

where $G_{v}$ is the gust factor for wind velocity defined by $\bar{V}_{\tau} / \bar{V}_{T}, \tau$ is the averaging time for gust velocity (e.g., $3-\mathrm{s}), T$ is the averaging time for wind-induced response calculation (e.g., 10min or 1-h) and $g_{v}$ is the peak factor for wind velocity. In AIJ, the gust factor $G_{q}$ is taken as unity because the mean wind velocity is used instead of the gust velocity.

\subsection{Across Wind and Torsional Loads}

In contrast to along wind loading where conventional gust factor approach is adopted among the codes, the approach to predict the across wind and torsional wind loadings were derived based on different concepts in each code. The partial loading approach, which just introduces the fractions on along wind 
loadings, is utilized in codes AS/NZS to address the across wind loadings. Further, wind tunnel derived data are widely adopted in wind design codes for the prediction of across wind and torsional loadings. Torsion is addressed in most of the codes by an applied moment derived by multiplying along wind loading by a defined eccentricity. Across-wind and torsional responses developed by wakeinduced effects can be dominant than in the along wind case for slender tall buildings. That is the main reason for higher degree of variation in across and torsional wind responses obtained through different codes/standards than in the case of the along wind response. This is further explained in the example sections later.

The nominal eccentricities specified in different standards are tabulated in Table 3. Only AJI code provides a detailed procedure for calculation of torsional moments. The effective eccentricity of the overall shear force is usually at least 5 to 10 percent of the building width, although it is often twice this and, in some cases, it can be much higher [12]. Therefore, current provisions available for prediction of torsional wind loading have to be further refined. Further, importance of development of these simplified provisions is well noted in many literature [12].

Table 3 - Minimum Eccentricity for Torsion

\begin{tabular}{|l|c|}
\hline Codes/Standards & Eccentricity (e/D) \\
\hline ASCE 7-05 & $3.5 \%$ \\
\hline BS EN 1991-1-4:2005 & $10 \%$ \\
\hline AS/NZS 1170.2:2011 & $20 \%$ \\
\hline
\end{tabular}

\subsection{Combination of Wind Loading}

Based on the total peak response, along wind, Across wind and torsional wind loads can be combined together in different ways. Different codes accompany different combination rationales. A partial loading approach with a load combination factor of 0.75 is adopted in American and Canadian standards. A root mean square method is adopted in Australian standard to combine along wind and across wind loads.

Japanese standard uses a constant factor (0.4) to combine along wind load with across wind and torsional loads. In addition, AIJ provides additional combinations considering a correlation coefficient $\left(\rho_{L T}\right)$ between the across wind and torsional loads. Further, review of several combination rules are reported in [13].

\section{Numerical Example}

Dynamic wind loading effects described in this paper is demonstrated through a numerical example in this section. Detailed discussion on dynamic wind loading calculated on a standard tall building (of which wind tunnel test results are reported [6]) consisting of forty-six stories and $183 \mathrm{~m}$ tall with plan dimensions of $30 \mathrm{~m} \times$ $46 \mathrm{~m}$ is considered. A conventional reinforced concrete structural system with central core and perimeter columns were considered. Figures 14 and 15 show floor system of the building and Finite Element Model developed, respectively.

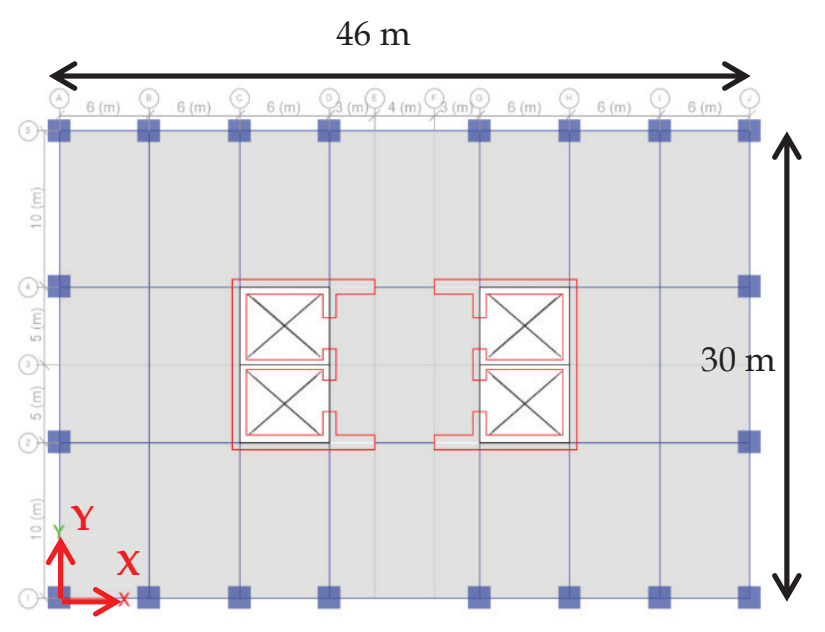

Figure 14 - Arrangement of Structural Elements in the Floor System

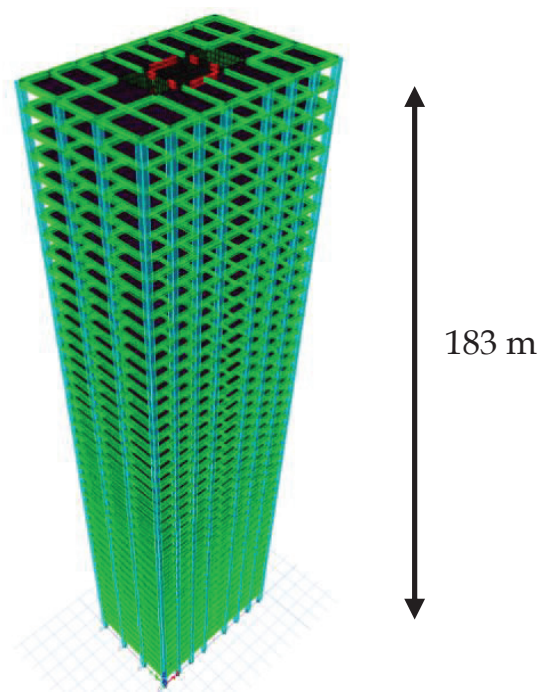

Figure 15 - Finite Element Model of the Example Building Considered

First three natural frequencies of the structure obtained from Finite element analysis are tabulated in Table 4. 
Table 4 - Dynamic Properties of the Building

\begin{tabular}{|c|c|}
\hline Mode & \multicolumn{1}{|c|}{ Frequency $(\mathrm{Hz})$} \\
\hline Mode 1 & 0.200 (Translation-Y) \\
\hline Mode 2 & $0.266($ Translation-X) \\
\hline Mode 3 & 0.350 (Rotation $-\mathrm{Z})$ \\
\hline
\end{tabular}

Dynamic wind loadings using four different wind codes were calculated for similar wind speeds adopted in the wind tunnel test (tabulated in Table 5).

Table 5 - Basic Wind Speeds at $10 \mathrm{~m}$ Height

\begin{tabular}{|c|c|}
\hline Averaging time & Wind speed $(\mathrm{m} / \mathrm{s})$ \\
\hline $3-\mathrm{sec}$ & 32 \\
\hline 10-min & 22 \\
\hline 1-hour & 20 \\
\hline
\end{tabular}

Table 6 - Terrain Categories Referred

\begin{tabular}{|l|l|}
\hline \multicolumn{1}{|c|}{ Code } & \multicolumn{1}{c|}{ Terrain Category } \\
\hline BS 6399-2 & open country near sea \\
\hline BSEN1991-1-4 & 0 - open/ coastal \\
\hline AS/NZS1170.2 & II - open/ few obstructions \\
\hline AIJ-RLB-2004 & II - open/ low vegetation \\
\hline
\end{tabular}

Variation of wind speed over the height of the building is an important factor that influences the calculated wind loading. Figure 16 presents the comparison of velocity profile factor (ratio between wind speed at specific height and reference wind speed at $10 \mathrm{~m}$ in open terrain), which represents the variation of wind velocity in the atmospheric boundary layer. This comparison clearly indicates significant deviations in the variation of wind speed multipliers predicted by different codes. These deviations may be the result of the different models adopted in the wind codes to define the velocity profile and associated parameters. Further, a close match in the variation of velocity profile for a range of terrain categories of AIJ-RLB-2004 and BS EN 1991-1-4 is observed. Wind speeds with different averaging periods adopted in each code may also contribute to this deviation in velocity profile.

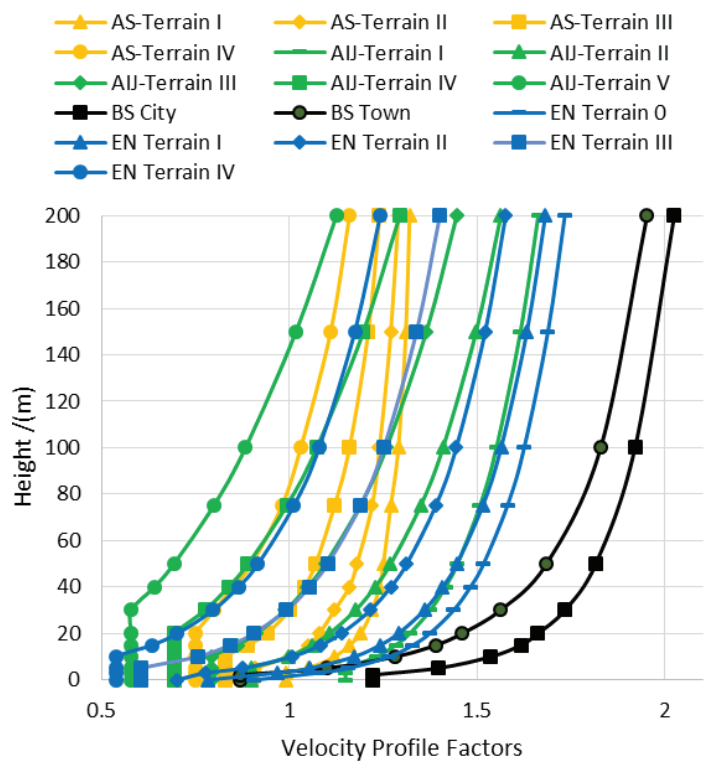

Figure 16 - Comparison of Velocity Profile Factors

An open terrain with minimum surface roughness (existing terrain category of relevant code that closely matches wind tunnel adopted velocity profile) was considered in this example. Based on the comparison of velocity profiles (in Figure 16) for different terrain categories specified in each code, terrain categories tabulated in Table 6 have been chosen. Wind speed variation over the building height predicted using wind codes for selected terrain categories and a velocity profile adopted in wind tunnel testing [14] are plotted in Figure 17. Wind loading was determined using four different wind codes, using relevant velocity profiles predicted by the code as well as unified profile adopted in the wind tunnel testing.

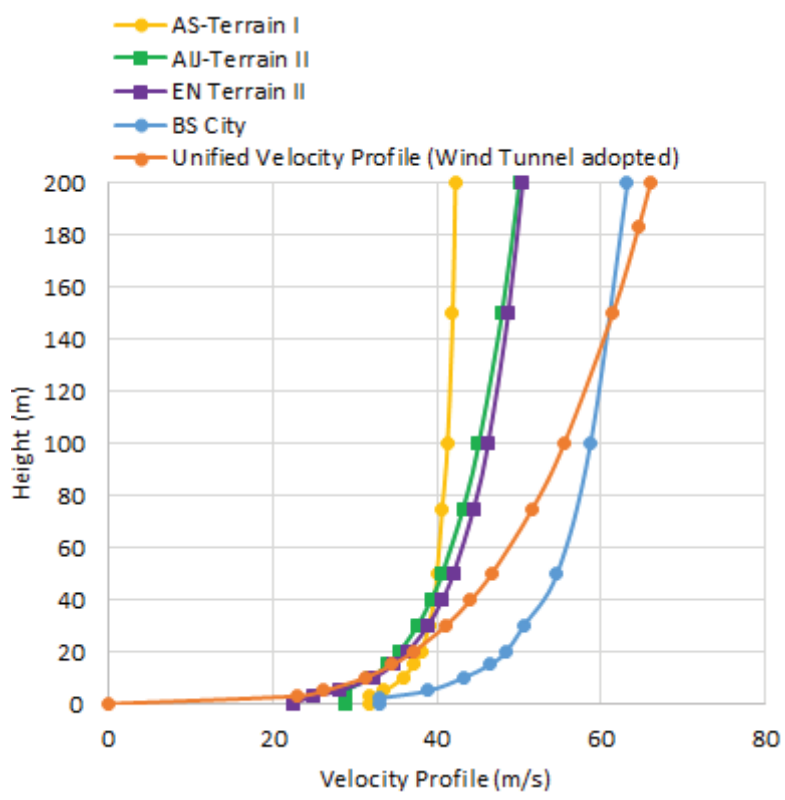

Figure 17 - Velocity Profiles Considered 
Wind speed and dynamic wind pressure at the top of the building, predicted by using both code based and unified velocity profiles, are tabulated in Table 7. Differences in wind speed and dynamic wind pressure clearly show that velocity profile is an important factor that determines the consistency of wind loading prediction using different wind codes. Comparison of wind loading effects presented below further emphasises this. Consequently, wind tunnel used velocity profile was used to compute dynamic wind loading predictions.

Table 7 - Comparison of Design Wind Speed and Dynamic Wind Pressure at the Top of the Structure

\begin{tabular}{|l|l|l|l|l|}
\hline Code & \multicolumn{2}{|l|}{$\begin{array}{l}\text { Wind speed } \\
(\mathrm{m} / \mathrm{s})\end{array}$} & \multicolumn{2}{l|}{$\begin{array}{l}\text { Dynamic pressure } \\
\text { at top (kPa) }\end{array}$} \\
\hline & Code & Unified & Code & Unified \\
\hline BS & 40 & 40 & 1.10 & 1.10 \\
\hline EN & 34 & 46 & 1.13 & 2.34 \\
\hline AS & 43 & 66 & 1.11 & 2.61 \\
\hline AIJ & 34 & 46 & 1.17 & 2.22 \\
\hline
\end{tabular}

Note: Wind speeds based on respective averaging time of the relevant code is referred

Comparison of along wind base moments estimated in two orthogonal wind directions are presented in Figures 18 and 19. Along wind base moments, determined from the wind codes except British code, are observed to be very closer to the wind tunnel estimation. British standard provides a lower estimate since wind induced responses are estimated over an averaging time of 60 minutes while other standards adopt 10 minutes as averaging time.

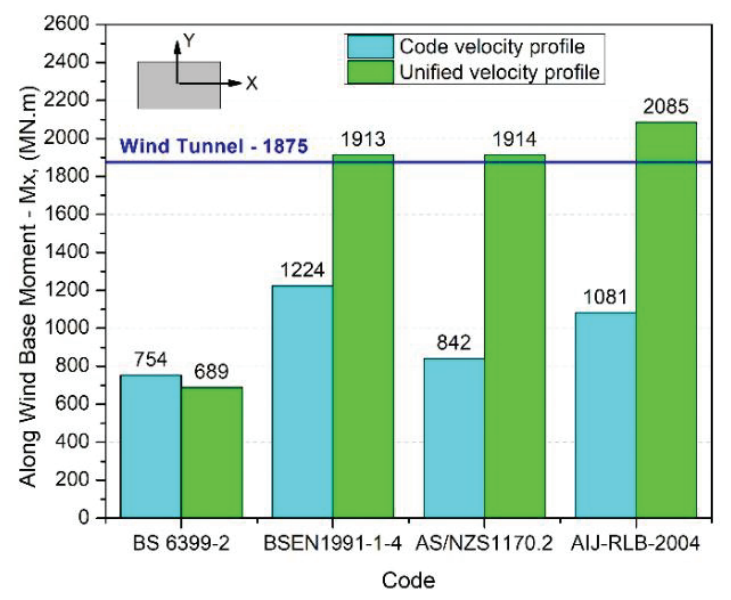

Figure 18 - Comparison of along Wind Base Moment - $M x$

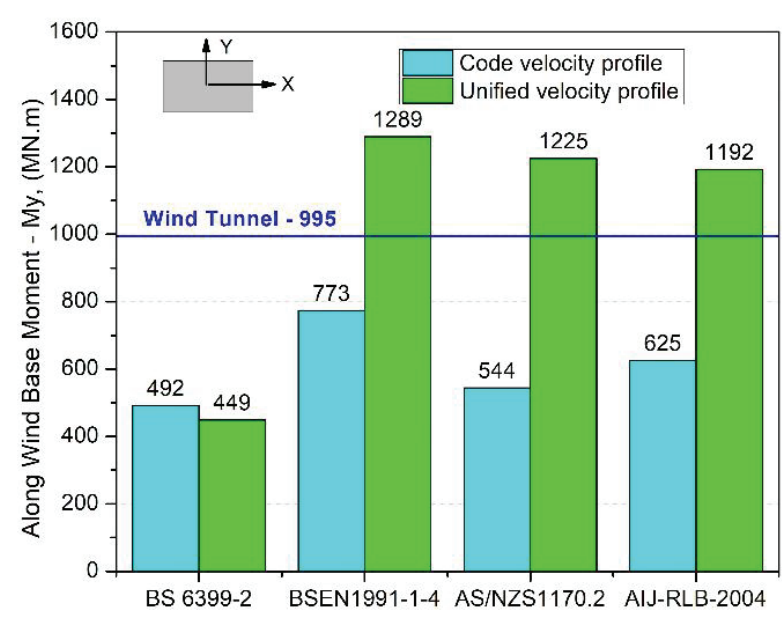

Figure 19 - Comparison of along Wind Base Moment - My

Figures 20 and 21 present comparison of across wind base moments estimated about both axes. Though across wind loading predicted by both Australian and Japanese standards well correlates with wind tunnel results for critical direction, Australian standard underpredicts across wind loading in other directions. Such inconsistency may be because Australian standard's equation uses breadth/ depth $(b / d)$ ratio, whereas Japanese standard uses depth/breath $(d / b)$ ratio, to determine across wind loading.

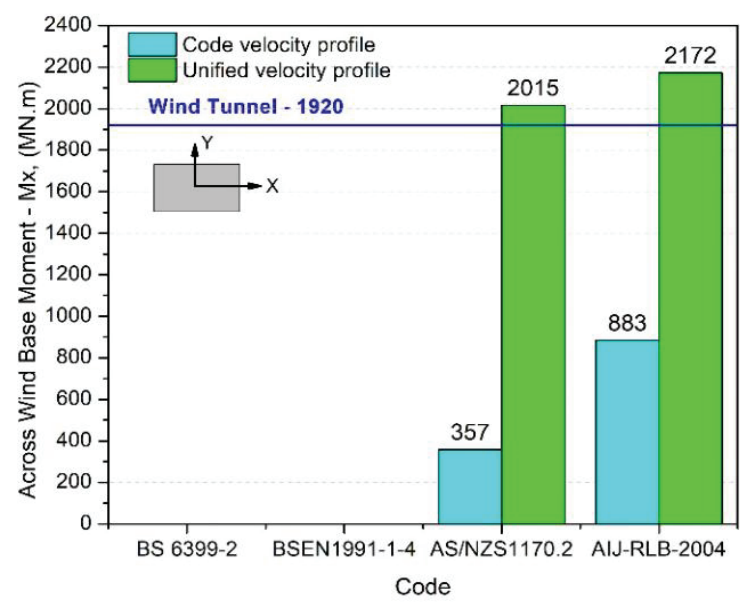

Figure 20 - Comparison of Across Wind Base Moment - $M x$ 


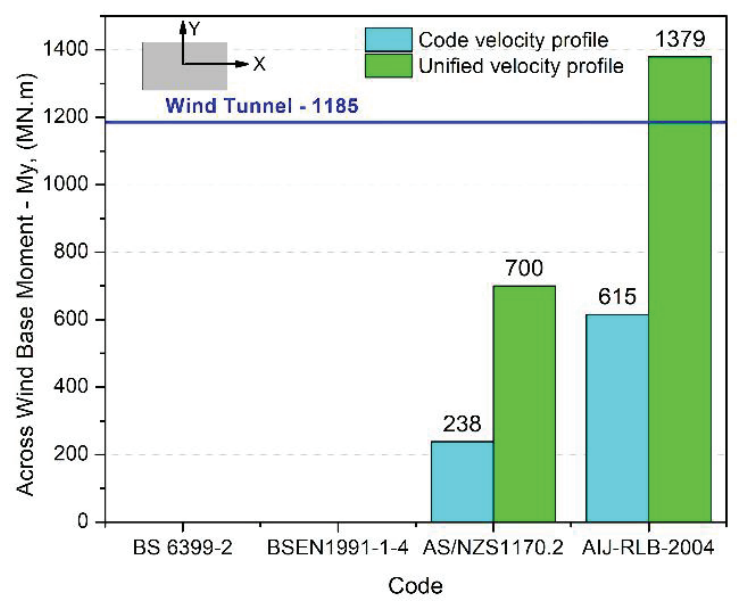

Figure 21 - Comparison of Across Wind Base Moments - My

Comparisons of along wind and across wind base moments graphically presented above are summarized in Table 8. All three wind codes except British standard provide a very consistent estimate of along wind base moment in both wind directions with a coefficient of variation of $4 \%$. The larger time averaging (as explained in Table 2) adopted in British standard yields a very lower estimate of base moment, thus it is ignored in the comparison. Further, estimated base moments using other standards well correlate with wind tunnel predictions for one principal axis where natural frequency of the example building $(0.200 \mathrm{~Hz})$ and tested building $(0.200 \mathrm{~Hz})$ is the same. Wind tunnel results significantly deviate for other axes due to slight variation in natural frequencies of model building $(0.266 \mathrm{~Hz})$ and tested building $(0.230 \mathrm{~Hz})$. Both Australian and Japanese standards provide a consistent across wind base moment estimate in critical direction with a coefficient of variation of $6 \%$, which also well correlates with wind tunnel results. However, a higher level of deviation with a coefficient of variation of $32 \%$ is observed for orthogonal wind direction. While Australian standard provides lower bound across wind base moment estimate, Japanese standard predictions are slightly conservative than wind tunnel test results. These discrepancies may have resulted due to contradictions found in the formulas adopted in both wind codes. Some of the contradictions will be summarized in later part of this paper.
Table 8 - Summary of Base Moment Comparison

\begin{tabular}{|l|r|r|r|r|}
\hline \multirow{2}{*}{ Code } & \multicolumn{2}{|c|}{$\begin{array}{c}\text { Along wind } \\
\text { base moment } \\
(\mathrm{MN} . \mathrm{m})\end{array}$} & \multicolumn{2}{c|}{$\begin{array}{c}\text { Across wind } \\
\text { base moment } \\
\text { (MN.m) }\end{array}$} \\
\cline { 2 - 5 } & \multicolumn{1}{|c|}{$M x$} & \multicolumn{1}{c|}{$M y$} & \multicolumn{1}{c|}{$M x$} & \multicolumn{1}{c|}{$M y$} \\
\hline BS 6399-2 & 689 & 449 & - & - \\
\hline BSEN1991-1-4 & 1913 & 1289 & - & - \\
\hline AS/NZS1170.2 & 1914 & 1225 & 2015 & 700 \\
\hline AIJ-RLB-2004 & 2056 & 1192 & 2172 & 1379 \\
\hline Mean & $\mathbf{1 9 6 1}$ & $\mathbf{1 2 3 5}$ & $\mathbf{2 0 9 4}$ & $\mathbf{1 0 4 0}$ \\
\hline Wind Tunnel & $\mathbf{1 8 7 5}$ & $\mathbf{9 9 5}$ & $\mathbf{1 9 2 0}$ & $\mathbf{1 1 8 5}$ \\
\hline $\begin{array}{l}\text { *Coefficient of } \\
\text { variation }\end{array}$ & $\mathbf{4} \%$ & $\mathbf{4} \%$ & $\mathbf{6 \%}$ & $\mathbf{3 2 \%}$ \\
\hline
\end{tabular}

* Mean and Coefficient of variation were calculated excluding BS 6399-2 predictions

Torsional moments were predicted as per the guidelines set out in three wind codes. Figures 22 and 23 show comparison of torsional moments determined for two approaching wind directions along both principal axes. A summary of the results is tabulated in Table 9 . In both wind directions, European code provides lower bound estimates well below the wind tunnel results, whereas Japanese standard estimates are vice versa. Australian standard provides reasonable estimates for wind approaching narrowest building face. However, for the wind approaching widest face of the building, estimated torsional moment is around twice of the actual. Lower bound results are obtained from the European code, because only partial wind loading from windward face is considered to determine the torsional wind load. Unlike other standards that simply depend on nominal eccentricities of windward face, Japanese code provides a formula to estimate the torsional wind loading. Consequently, difference between torsional moments developed for two orthogonal wind directions is relatively less compared to other standards. Further, this comparison shows high level of inconstancies (with coefficient of variation exceeding more than $60 \%$ ) found in provisions adopted by wind codes for the estimation of torsional loading. As a result, it is seen that existing wind code provisions are capable to predict along wind loading more precisely and across wind loadings to a certain level of accuracy. However, reliability of code provisions to estimate torsional wind loading is questionable. 


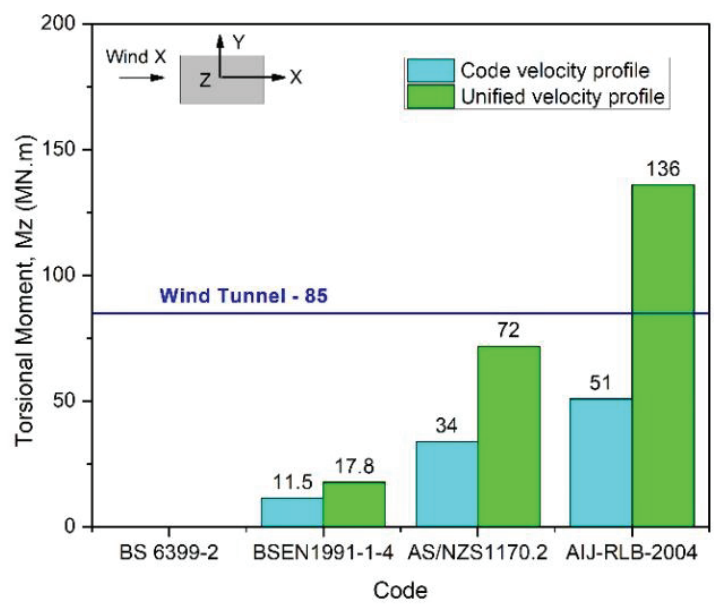

Figure 22 - Comparison of Torsional Moments

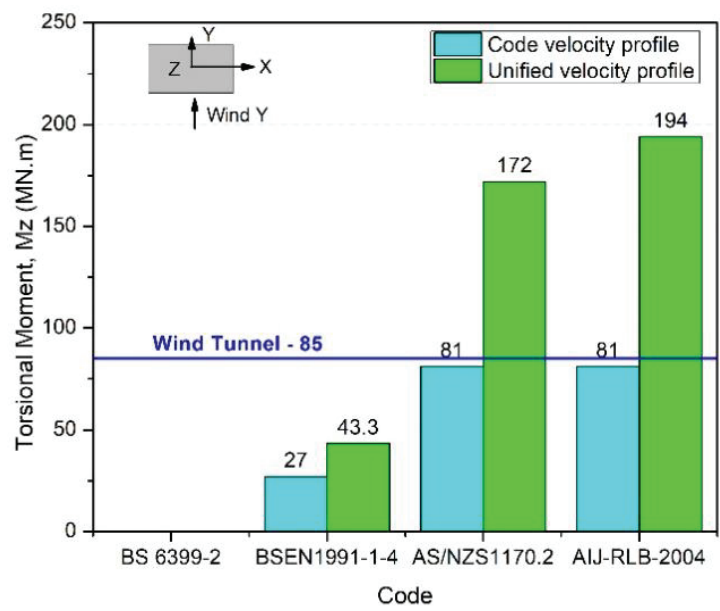

Figure 23 - Comparison of Torsional Moments

Table 9 - Summary of Torsional Moment Comparison

\begin{tabular}{|l|c|c|}
\hline \multirow{2}{*}{ Code } & \multicolumn{2}{|l|}{$\begin{array}{l}\text { Torsional } \\
\text { Moment }-M z \text { (MN.m) }\end{array}$} \\
\cline { 2 - 3 } & X - Wind & Y - Wind \\
\hline BS 6399-2 & - & - \\
\hline BSEN1991-1-4 & 18 & 39 \\
\hline AS/NZS1170.2 & 72 & 172 \\
\hline AIJ-RLB-2004 & 136 & 194 \\
\hline Mean & $\mathbf{7 5}$ & $\mathbf{1 3 5}$ \\
\hline Wind Tunnel & $\mathbf{8 5}$ & $\mathbf{8 5}$ \\
\hline $\begin{array}{l}\text { *Coefficient of } \\
\text { variation }\end{array}$ & $\mathbf{7 8 \%}$ & $\mathbf{6 2 \%}$ \\
\hline
\end{tabular}

Contribution of mean, background and resonance components as a portion of total along wind base moment is presented in Figures 24 and 25. It is noticed that base moment predicted by British standards closely matches mean (hourly) base moment derived from the wind tunnel test. As described in Table 2, other three standards estimate the wind responses averaged over 10 minutes, subsequently providing higher mean component compared to the British standard.
Simplified dynamic augmentation factor, derived based on the building height adopted in British standard, does not allow to determine background and resonance components individually. Due to the differences in averaging time of wind responses, excluding British standard, other three standards consist of approximately $60 \%, 30 \%$ and $10 \%$ of mean, background and resonance components, respectively, in the along wind loading of building considered. This highlights the importance of dynamic wind loading effects represented by background component (due to the turbulence in wind stream) and resonance component (due to dynamic behaviour of the structure) in addition to the mean wind loading.

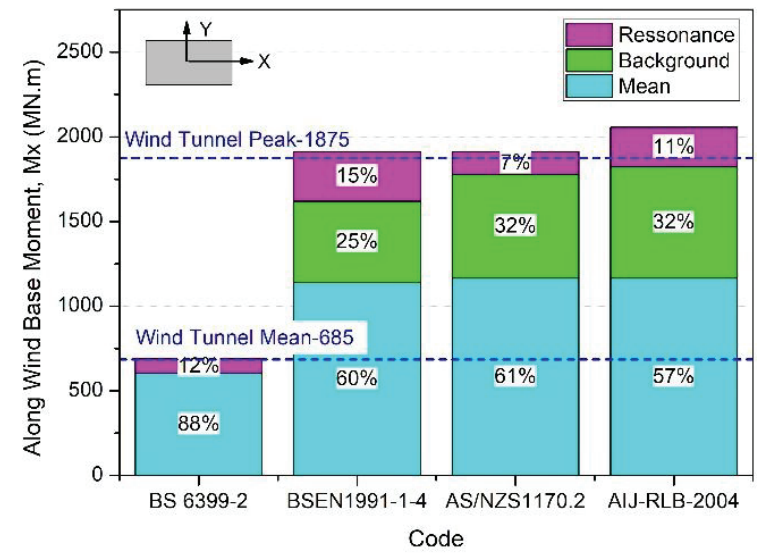

Figure 24 - Contribution of Mean, Background and Resonance Components in Base Moment $-M x$

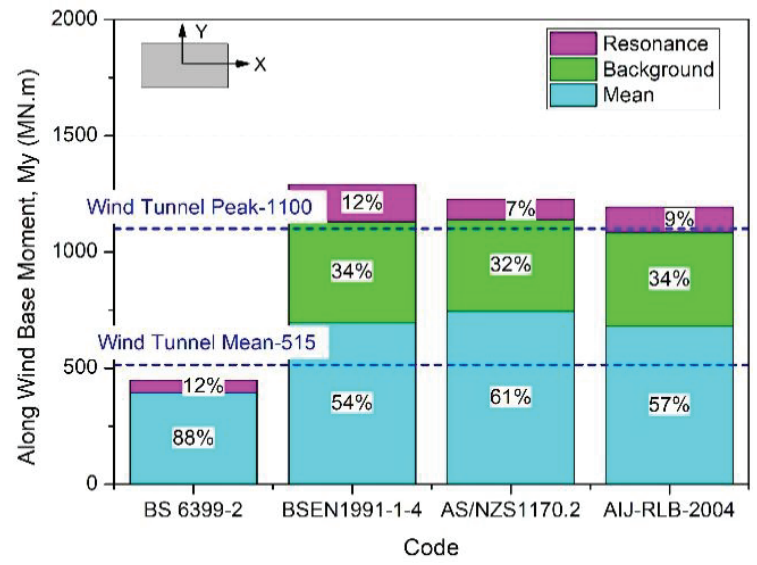

Figure 25 - Contribution of Mean, background and Resonance Components in along Wind Base Moment - My

Dynamically induced wind effects such as across wind dynamic moment and torsional wind moment comprise both background and resonance components. Contribution of background and resonance components as a percentage of total across wind base moments is presented in Figures 26 and 27. Reduction in the resonance component, when loading arises 
in rigid building axis (2nd modal direction) compared to flexible axis (1st modal direction), can be observed from the results obtained from both AS and AIJ standards. However, AIJ standard yields reasonable predictions that match wind tunnel results in both wind directions, due to the consideration of background contribution. Australian standard neglects background contribution in the across wind loading. Consequently, it may result in under prediction of across wind loading in some cases as illustrated. Further, across wind spectrum coefficient derived based on the reduced velocity is adopted in the AS standard. In contrast, AIJ uses a correlation coefficient taking into account building mass and mode shape in the across wind loading formula.

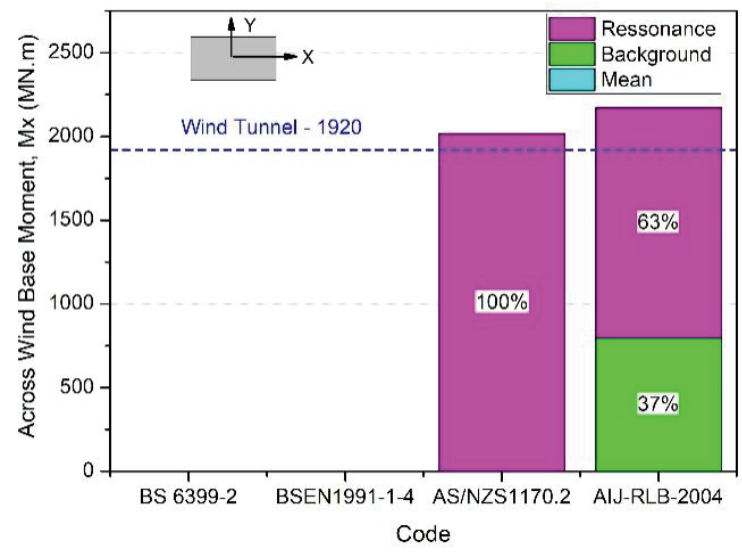

Figure 26 - Contribution of Mean, Background and Resonance Components in across Wind Base Moment - $M x$

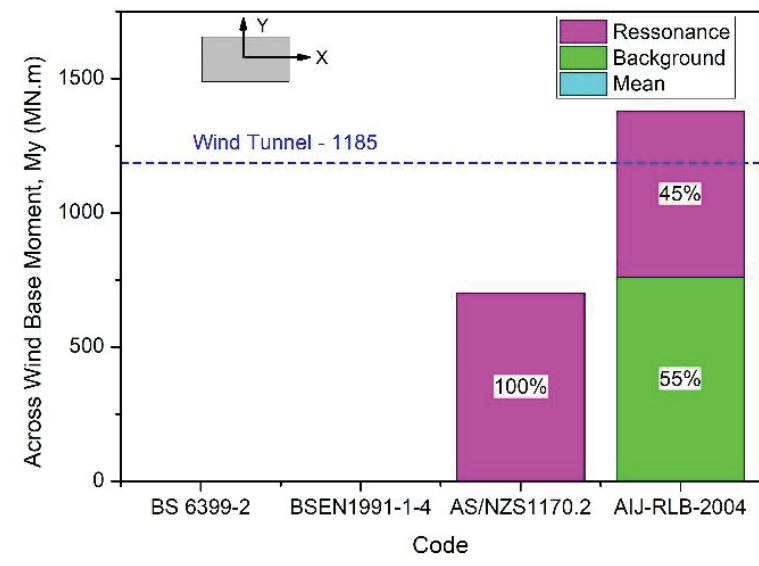

Figure 27 - Contribution of Mean, Background and Resonance Components in Across Wind Base Moment - My

Contributions of background and resonance components as a percentage of total torsional base moments are presented in Figures 28 and 29 , respectively. As descried earlier in this paper, only AIJ standard provides detailed formula identifying the resonance and background components separately, whereas other standards specify a nominal eccentricity.

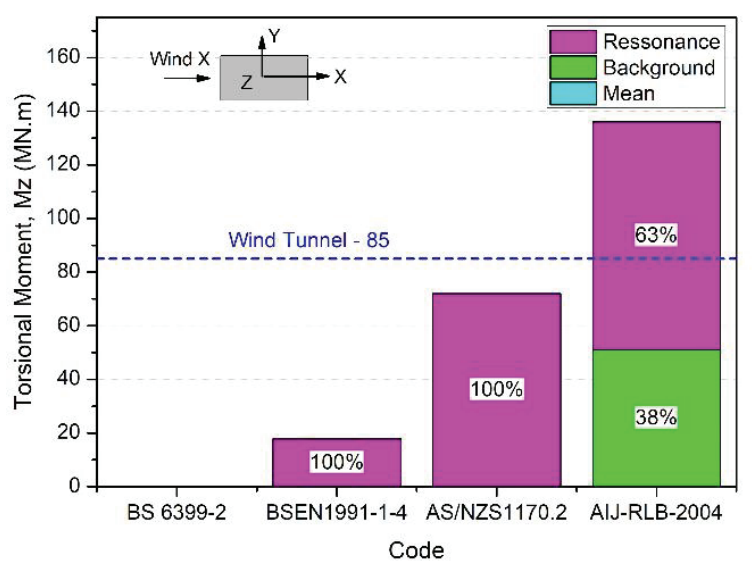

Figure 28 - Contribution of Mean, Background and Resonance Components in Torsional Wind Base Moment - Mz

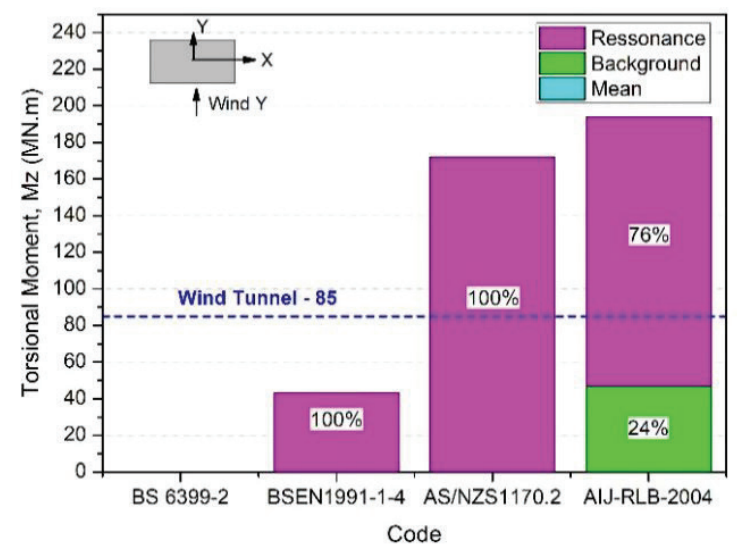

Figure 29 - Contribution of Mean, Background and Resonance Components in Torsional Wind Base Moment - Mz

Figures 30 to 33 present the comparison of both along wind and across wind base shears with the proportion of mean, background and resonance components. This comparison clearly explains that crosswind loading is highly influenced by resonance response which depends on the dynamic characteristics of the building compared to along wind loading.

Comparison of base shear results are summarized in Table 8. Same explanation of base moments presented in this paper can be extended to elaborate the trend of variation in base shear predictions.

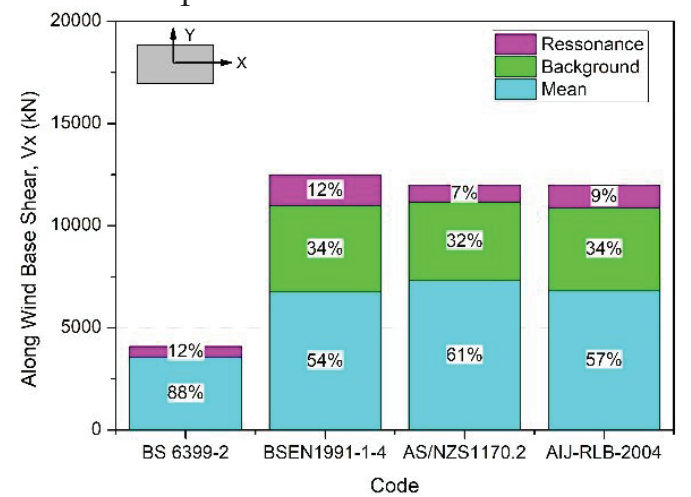

Figure 30 - Mean, Background and Resonance Components in along Wind Base Shear $-V x$ 


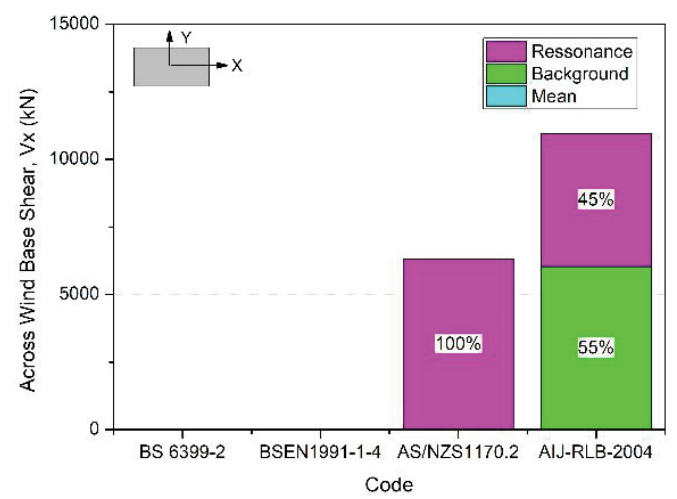

Figure 31 - Mean, Background and Resonance Components in across Wind Base Shear $-V x$

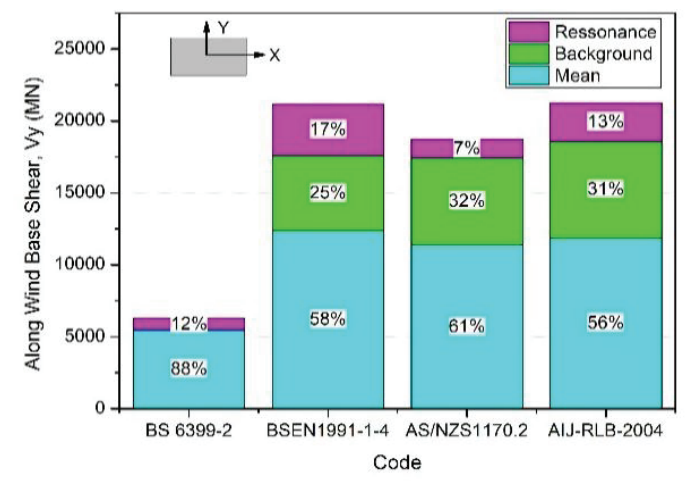

Figure 32 - Mean, Background and Resonance Components in along Wind Base Shear - Vy

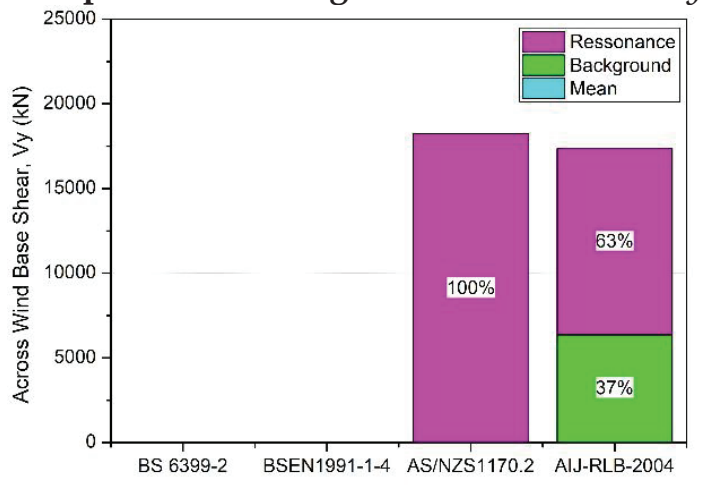

Code

Figure 33 - Mean, Background and Resonance Components in across Wind Base Shear - Vy

Table 10 - Summary of Base Shear Comparison

\begin{tabular}{|l|r|r|r|r|}
\hline \multirow{2}{*}{ Code } & \multicolumn{2}{|l|}{$\begin{array}{l}\text { Along wind } \\
\text { Base Shear } \\
(\mathrm{MN})\end{array}$} & \multicolumn{2}{l}{$\begin{array}{l}\text { Cross wind } \\
\text { Base Shear } \\
(\mathrm{MN})\end{array}$} \\
\cline { 2 - 5 } & $F x$ & $F y$ & $F x$ & $F y$ \\
\hline BS 6399-2 & 4.08 & 6.26 & - & - \\
\hline BSEN1991-1-4 & 12.49 & 19.02 & - & - \\
\hline AS/NZS1170.2 & 12.00 & 18.74 & 6.32 & 18.22 \\
\hline AIJ-RLB-2004 & 12.00 & 21.26 & 10.96 & 17.34 \\
\hline $\begin{array}{l}\text { *Coefficient of } \\
\text { variation }\end{array}$ & $\mathbf{2} \%$ & $\mathbf{7 \%}$ & $\mathbf{3 8 \%}$ & $\mathbf{3 \%}$ \\
\hline
\end{tabular}

* Coefficient of variations were calculated excluding BS 6399-2 predictions
Natural frequency of the structure and damping ratio are two important properties that control the resonance component of dynamic wind loading on a structure. Influence of the damping ratio and natural frequency of the structure on both along and across wind base moments are presented in Figures 34 and 35 , respectively. Since a simplified dynamic augmentation factor based on the building height is adopted in the British code to represent the dynamic wind loading, its predictions are not reflecting either building frequency or damping. Discrepancies between other three code predictions are observed to decrease with increased damping for both along wind and across wind loading. Further, it is noted that, across wind loading becoming more dominant in low damping region as expected. Figure 35 shows that except British standard other three standards provide consistence along wind loading prediction. Influence of frequency in the wind loading is observed to be negligible for rigid structures where frequency is greater than $1 \mathrm{~Hz}$. Further, when frequency is less than $1 \mathrm{~Hz}$, along wind loading gradually increases due to the increment of resonance component, and a drastic increment is observed beyond $0.25 \mathrm{~Hz}$. Across wind loading prediction by AIJ standard and AS standard are consistent in a narrow frequency range closer to $0.20 \mathrm{~Hz}$. Extrapolating AS code provisions for dynamically sensitive structures with frequency less than $0.20 \mathrm{~Hz}$ leads to incorrect across wind estimates where as AIJ provisions show drastic increment of across wind response closer to vortex shedding frequency as anticipated. In higher frequency range, AIJ standard across wind loading predictions are more than twice the AS standard estimates.

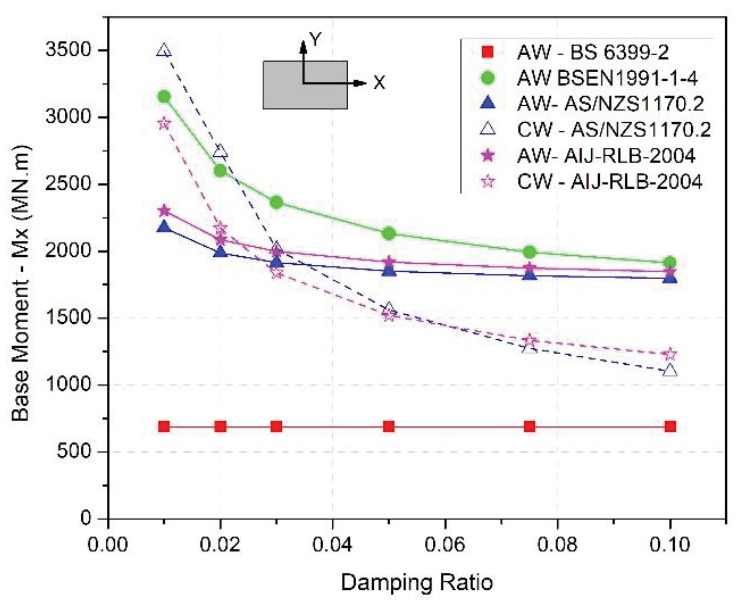

Figure 34 - Influence of Damping Ratio in the Base Moment 


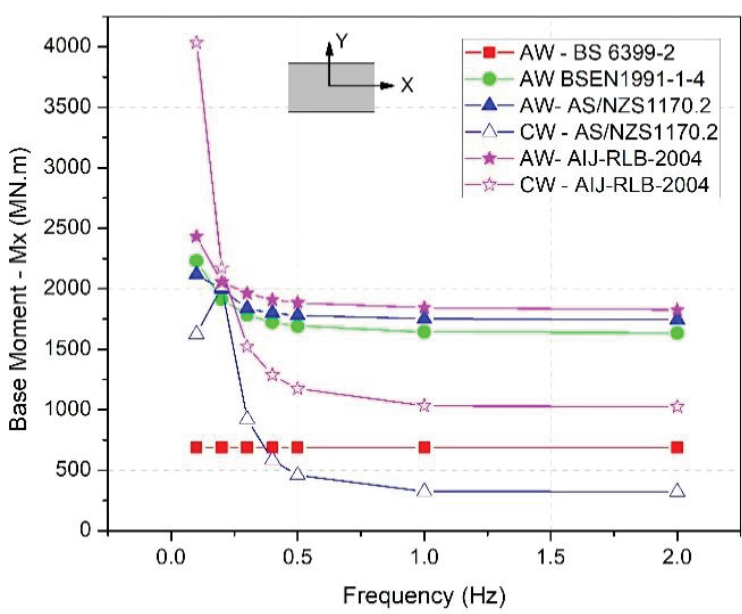

Figure 35 - Influence of Structure's Natural Frequency in the Base Moment

In order to examine the impact of wind loading and the directional combinations derived using different wind codes, axial loading on a corner column and axial and shear stresses on a critical location of the core wall under wind loading, are extracted from the Finite element analysis and tabulated in Table 9. Wind induced axial loads estimated from the British standard are found to be very low compared to other standards since it is based on mean wind loading and across wind loading is ignored. Further, in the absence of a method to determine torsional moments, torsional shear stresses in the core wall are under estimated. Ignorance of across wind loading leads to relatively low axial loading of European standard estimate. Further, relatively less torsional wind loading estimate results in under estimation of torsional shear stresses. Though AIJ and AS standards provide fairly consistent results, significant deviations are observed resulting from inconsistencies found in their across wind and torsional wind loading and method of combining different wind loading components (along wind, across wind and torsion). It can be noted that, due to various reasons, huge differences are observed in wind induced internal force demands developed in structural elements predicted by different codes. However, structural capacities are generally designed satisfactorily without intention, due to conservativeness in design such as safety factor on loading and materials.
Table 11 - Characteristic Loads/ Stress under Critical Load Case for Wind Against Widest Face

\begin{tabular}{|l|c|c|c|}
\hline Code & $\begin{array}{l}\text { Column } \\
\text { Load } \\
(\mathrm{kN})\end{array}$ & $\begin{array}{l}\text { Wall } \\
\text { Axial } \\
\text { Stress } \\
(\mathrm{MPa})\end{array}$ & $\begin{array}{l}\text { Wall } \\
\text { Shear } \\
\text { Stress } \\
(\mathrm{MPa})\end{array}$ \\
\hline BS 6399-2 & 1004 & 2.39 & 0.39 \\
\hline BSEN1991-1-4 & 2789 & 6.64 & 1.46 \\
\hline AS/NZS1170.2 & 3420 & 8.06 & 2.37 \\
\hline AIJ-RLB-2004 & 3807 & 9.04 & 2.44 \\
\hline Gravity Load* & 6900 & 7.80 & - \\
\hline
\end{tabular}

\section{Conclusions}

This study presents an overview of existing wind codes and their applications to the dynamic wind loading calculations for tall buildings. The fundamentals of dynamic wind loading and available provisions in four international standards commonly adopted in Sri Lanka were discussed. Finally, a numerical comparison is used to demonstrate the dynamic wind loading and interpretation of different wind codes in numerical calculations. Comparisons of estimated wind loadings were presented along with wind tunnel test results to highlight accuracy of existing code predictions. Estimated wind induced forces in critical members of lateral load resistance system of a building were presented to emphasise the importance of precise estimation of wind loading in tall building design.

BS 6399 standard adopts hourly mean along wind loads with a simplified dynamic augmentation factor that accounts for dynamic effects where as BSEN1991-1-4, AS/NZS1170.2 and AIJ-RLB-2004 adopt wind loading averaged over a short time period of 10 minutes. This results in a very low wind loading prediction by BS 6399. Further, dynamic properties such as frequency and damping are not included in the dynamic wind loading calculation of BS 6399. Guest factor method adopted in BSEN1991-1-4, AS/NZS1170.2 and AIJ-RLB-2004A provides a very consistent along wind loading estimate that correlates with wind tunnel predictions. Effect of building frequency and damping in resonance response is clearly interpreted in these three standards. Further, it was noted that along wind loading of the building considered comprises mean, background and resonance contributions approximately $60 \%, 30 \%$ and $10 \%$, respectively. Resonance component is 
observed to be negligible when structural frequency is higher than $1 \mathrm{~Hz}$. When frequency is less than $1 \mathrm{~Hz}$, along wind loading is observed to be gradually increasing due to the increment of resonance component and a drastic increment is observed beyond $0.25 \mathrm{~Hz}$.

Across wind loading prediction by AIJ standard and AS standard are consistent in a narrow frequency range closer to $0.20 \mathrm{~Hz}$. Extrapolating AS code provisions for dynamically sensitive structures with frequency less than $0.20 \mathrm{~Hz}$ leads to wrong across wind estimates, whereas AIJ provisions show drastic increment of across wind response closer to vortex shedding frequency as anticipated. However, higher level of deviation is observed for some wind directions between AIJ and AS/NZS standards in the prediction of across wind loading. These discrepancies may have resulted due to contradictions found in the formulas adopted in both wind codes. Further, background contribution is ignored in AS/NZS standard. In addition, a cross wind spectrum coefficient derived based on the reduced velocity is adopted in the AS/NZS standard. In contrast, AIJ uses a correlation coefficient taking into account building mass and mode shape in the across wind loading formula.

Consideration of partial application of windward face loading in BSEN1991-1-4 in the calculation of torsional loading results in lower bound torsional moment. AS/NZS1170.2 recommends a nominal eccentricity of $20 \%$ of the windward face width, which provides a conservative estimate when wind is approaching against widest face of the building. Unlike other standards which simply rely on nominal eccentricities, Japanese code provides a formula to estimate the torsional wind loading. Consequently, difference between torsional moments developed for two orthogonal wind directions is relatively less compared to other standards. However, for the wind approaching towards widest face of the building, estimated torsional moment is around twice the actual.

Based on the comparison it is noted that, existing wind code provisions are capable to predict along wind loading more precisely and across wind loadings to a certain level of accuracy. However, reliability of code provisions to estimate torsional wind loading is questionable.
Based on the study it was noted that, due to various reasons, significant differences are observed in wind induced internal force demands developed in structural elements predicted by different codes. However, structural capacities are generally designed satisfactorily without intention to be conservative in design such as requirements of higher stiffness for serviceability and habitability, and application of safety factors on loading and materials. In modern design codes safety factors are compromised and performance based wind design is adopted to arrive at optimum design. Thus, more attention should be paid in the precise estimation of wind loading and its directional combinations in order to ensure safety.

\section{Recommendation for Further work}

Based on this study it was noted that a strong requirement exists to harmonize the existing wind code provisions for prediction of across wind and torsional wind loading. Further, directional combinations proposed in current wind codes need to be further refined as they influence the internal forces on structural elements of the building.

\section{References}

1. Kijewski, T., and Kareem, A., 1998 "Dynamic Wind Effects: A Comparative Study of Provisions in Codes and Standards with Wind Tunnel Data" Wind Struct. An Int. J.1 77-109.

2. Boggs, D., and Dragovich, J., "The Nature of Wind Loads and Dynamic Response" Aci Sp240SP 240 15-44, 2006.

3. Nakai, M., Hirakawa, K., Yamanaka, M., Okuda, H., and Konishi, A.,"PerformanceBased Wind-Resistant Design for High-Rise Structures in Japan" Int. J. High-Rise Build.2 271-83,2013.

4. Holmes, J., Tamura, Y., and Krishna, P., "Comparison of Wind Loads Calculated by Fifteen different Codes and Standards, for Low, Medium and High-Rise Buildings" 11th Am. Conf. Wind Eng,2009.

5. Kwon, D. K., and Kareem, A., "Comparative Study of Major International Wind Codes and Standards for Wind Effects on Tall Buildings" Eng. Struct.51 23-35, 2013. 
6. Melbourne, W. H., "Comparison of Measurements on the CAARC Standard Tall Building Model in Simulated Model Wind Flows" J. Wind Eng. Ind. Aerodyn.6 7388,1980 .

7. Taranath, B. S., Reinforced Concrete Design of Tall Buildings, 2009.

8. Choi, E. C. C., Proposal For Unified Terrain Categories Exposures, 2009.

9. Stathopoulos, T., Zisis, I., and Wang, K., "Terrain Classification and Exposure Factor in the National Building Code of Canada", 2005.

10. Ge, Y., Jin, X., and Cao, S., "Comparison of APEC Wind Loading Codification and Revision of Chinese National Code," 2005.

11. Davenport, A. G., "The Treatment of Wind Loading on Tall Buildings" J Struct Div, ASCE 11-34,1967.

12. Boggs, D. W., Hosoya, N., and Cochran, L., "Sources of Torsional Wind Loading on Tall Buildings: Lessons from the Wind Tunnel", 2004.

13. Tamura, Y., Kim, Y. C., and Kikuchi, H., "Correlation and Combination of Wind Force Components and Responses" 6th Eur. African Conf. Wind Eng. EACWE 2013125 81-93, 2013.

14. Holmes, J. D., and Tse, T. K. T., "International High-Frequency Base Balance Benchmark Study", 2014.

15. Architectural Institute of Japan (AIJ). "RLB Recommendations for Loads on Buildings. Tokyo (Japan)": Structural Standards Committee, Ar-chitectural Institute of Japan; 2004.

16. Joint Technical Committee. "AS/NZS 1170.2:2011 Structural design actions - Part 2: Wind actions. Australian/ New Zealand Standard (AS/NZS)": Joint Technical Committee BD-006, Australia/ New Zealand; 2011.

17. European Committee for Standardization (CEN). Eurocode 1: "Actions on structures Part 1-4: General actions - wind actions". EN 1991-1-4:2005/ AC: 2010 (E). Europe: European Standard (Eurocode), European Committee for Standardization (CEN): 2010.

18 BS6399-1. (1996). "Loading for Buildings.

British Standards Institution". 